\title{
A Generalized Uncertain Fractional Forward Difference Equations of Riemann-Liouville Type
}

\author{
Pshtiwan Othman Mohammed ${ }^{1}$ \\ ${ }^{1}$ Department of Mathematics, College of Education, University of Sulaimani, Sulaimani, Kurdistan Region, Iraq \\ Correspondence: Pshtiwan Othman Mohammed, Department of Mathematics, College of Education, University of Su- \\ laimani, Sulaimani, Kurdistan Region, Iraq. E-mail: pshtiwansangawi@gmail.com
}

Received: June 20, 2019 Accepted: July 13, 2019 Online Published: July 23, 2019

doi:10.5539/jmr.v11n4p43～URL: https://doi.org/10.5539/jmr.v11n4p43

\begin{abstract}
In this paper, we firstly recall the definition of an uncertain fractional forward difference equation with Riemann-Liouvillelike forward difference. After that analytic solutions to a generalized uncertain fractional difference equations are solved by using the Picard successive iteration method. Moreover, the existence and uniqueness theorem of the solutions are proved by applying Banach contraction mapping theorem. Finally, two examples are presented to illustrate the validity of the existence and uniqueness theorem.
\end{abstract}

Keywords: Riemann-Liouville fractional integral, discrete fractional calculus, uncertainty theory, fractional forward difference equations

\section{Introduction}

Fractional calculus and fractional differential equations are rising fields recently drawing attention from both applied and theoretical disciplines. During the last two decades, they have been successfully applied to several fields (Miller, K. S., \& Ross, B. 1993; Podlubny, I. 1999; Kilbas, A. A., Srivastava, H. M., \& Trujillo, J. J. 2006; Diethelm, K. 2010; Hamasalh F. K., \& Mohammed, P. O. 2015a; Hamasalh F. K., \& Mohammed, P. O. 2015b). There are many interested researches on what is usually called integer-order difference equations; see (Agarwal, R. P. 2000; Bohner, M., \& Peterson, A. C. 2003). Discrete fractional calculus and fractional difference equations represent a new area for scientists which were developed slowly in their early stages. Some works are dedicated to boundary value problems, initial value problems, chaos and stability for the fractional difference equations (Atici F., \& Eloe, P. 2007; Atici F., \& Eloe, P. 2009; Goodrich, C. 2011; Goodrich, C., \& Peterson, A. 2015; Wu, G., \& Baleanu, D. 2015; Wu, G., Baleanu, D., \& Luo, W. 2017; Wu, G., Baleanu, D., \& Zeng, S. 2018).

Recently, uncertain fractional differential and difference equations have been introduced and investigated in order to model the continuous or discrete systems with memory effects and human uncertainty by (Zhu, Y. 2015a; Zhu, Y. 2015b; Lu, Z., \& Zhu, Y. 2018; Lu, Z., \& Zhu, Y. 2019; Lu, Q., Zhu, Y., \& Lu, Z. 2019). Furthermore, existence of the solution to uncertain fractional differential equation was discussed by Zhu, Y. (2015a). Also, an existence and uniqueness theorem of solutions to a special type of UFFDEs is presented by Lu, Q., Zhu, Y., \& Lu, Z. (2019). Meanwhile, Lu, Z., \& Zhu, Y. (2019) have been created the relations between uncertain fractional differential equations and the associated fractional differential equations via the comparison theorems for fractional differential equations of Caputo type by Lu, Z., \& Zhu, Y. (2018).

Motivated by the works mentioned above, we will define a generalized uncertain fractional forward difference equation (GUFFDE) and present an existence and uniqueness theorem of solutions to a special type of GUFFDEs.

\section{Preliminaries}

In this section, we recall some important facts in uncertainty theory; see for more details (Atici F., \& Eloe, P. 2007; Atici F., \& Eloe, P. 2009; Goodrich, C. 2011; Goodrich, C., \& Peterson, A. 2015; Wu, G., \& Baleanu, D. 2015; Wu, G., Baleanu, D., \& Luo, W. 2017; Wu, G., Baleanu, D., \& Zeng, S. 2018; Zhu, Y. 2015a; Zhu, Y. 2015b; Lu, Z., \& Zhu, Y. 2018; Lu, Z., \& Zhu, Y. 2019; Lu, Q., Zhu, Y., \& Lu, Z. 2019). Let us denote by $\mathcal{F}_{\mathcal{D}}$ the set of real valued functions defined on $\mathcal{D}$. Let $\mathbb{N}_{b}=\{b, b+1, b+2, \ldots\}$ for $b \in \mathbb{R}$ and $\sigma(r):=r+1$ for $r \in \mathbb{N}_{b}$.

Definition 2.1 (Holm, M. (2011)). For a function $p: \mathbb{N}_{b} \rightarrow \mathbb{R}$ the forward difference operator is defined as:

$$
\Delta p(\mathbf{z})=p(\sigma(\mathbf{z}))-p(\mathbf{z}), \quad \mathbf{z} \in \mathbb{N}_{b},
$$


while the difference sum is given by

$$
\Delta_{b}^{-1} p(\mathbf{z})=\sum_{r=b}^{\mathbf{z}-1} p(r), \quad \mathbf{z} \in \mathbb{N}_{b}
$$

Definition 2.2 (Atici F., \& Eloe, P. 2007; Holm, M. 2011). For arbitrary $t, v \in \mathbb{R}$ the $h$-factorial function is defined by:

$$
\begin{aligned}
\mathbf{z}^{(v)} & =\frac{\Gamma(\mathbf{z}+1)}{\Gamma(\mathbf{z}+1-v)}, \\
\mathbf{z}^{(0)} & =1,
\end{aligned}
$$

where $\Gamma$ is the well-known Euler gamma function, and we use the convention that division at a pole yields zero.

Definition 2.3 (Atici F., \& Eloe, P. 2007; Holm, M. 2011). Let $p \in \mathcal{F}_{\mathcal{D}}$. The fractional $h$-sum of order $v>0$ is given by :

$$
\begin{aligned}
\Delta_{b}^{-v} p(\mathbf{z}) & =\frac{1}{\Gamma(v)} \sum_{r=b}^{\mathbf{z}-v}(\mathbf{z}-\sigma(r))^{(v-1)} p(r), \\
\Delta_{b}^{0} p(\mathbf{z}) & =p(\mathbf{z}),
\end{aligned}
$$

where $b \in \mathbb{R}, \sigma(r)=r+1$, and $\Delta_{b}^{-v} p(\mathbf{z})$ is defined on $\mathbb{N}_{b+v}$.

Lemma 2.1 (Atici F., \& Eloe, P. 2007; Holm, M. 2011). Let $p \in \mathcal{F}_{\mathcal{D}}$ and $v, \vartheta>0$, then we have:

$$
{ }_{b+\vartheta} \Delta^{-v}\left[\Delta_{b}^{-\vartheta} p(\mathbf{z})\right]=\Delta_{b}^{-(\vartheta+v)} p(\mathbf{z})={ }_{b+v} \Delta^{-\vartheta}\left[\Delta_{b}^{-v} p(\mathbf{z})\right]
$$

for $\mathbf{z} \in \mathbb{N}_{b+(v+\vartheta)}$.

Lemma 2.2 (Holm, M. (2011)). Let $p \in \mathcal{F}_{\mathcal{D}}$ and $m$ be a positive integer with $0<m-1<\vartheta \leq m$, then the RiemannLiouville-like fractional forward difference is given by:

$$
\Delta_{b}^{\vartheta} p(\mathbf{z})=\Delta^{m}\left(\Delta_{b}^{-(m-\vartheta)} p(\mathbf{z})\right) .
$$

Lemma 2.3 (Holm, M. (2011)). Let $p \in \mathcal{F}_{\mathcal{D}}$ and $v \geq 0$, then we have:

$$
\Delta_{b}^{-v} \Delta p(\mathbf{z})=\Delta \Delta_{b}^{-v} p(\mathbf{z})-\frac{(\mathbf{z}-b)^{(v-1)}}{\Gamma(v)} p(b)
$$

for $\mathbf{z} \in \mathbb{N}_{b+v}$.

Lemma 2.4 (Bastos, N. R. O. et al (2011)). Let $b, v \in \mathbb{R}$. Then for each $\mathbf{z} \in \mathbb{N}_{b+v}$, we have

$$
\Delta_{b}\left(\frac{(\mathbf{z}-b)^{(v)}}{\Gamma(v+1)}\right)=\frac{(\mathbf{z}-b)^{(v-1)}}{\Gamma(v)} .
$$

Lemma 2.5 (Holm, M. (2011)). Let $p \in \mathcal{F}_{\mathcal{D}}, v \in \mathbb{R}$ and $q$ is any positive integer, then

$$
\Delta_{b}^{-v} \Delta^{q} p(\mathbf{z})=\Delta^{q} \Delta_{b}^{-v} p(\mathbf{z})-\sum_{k=0}^{q-1} \frac{(\mathbf{z}-b)^{(v-q+k)}}{\Gamma(v-q+k+1)} \Delta^{k} p(b)
$$

for $\mathbf{z} \in \mathbb{N}_{b+v}$.

Lemma 2.6 (Holm, M. (2011)). Suppose that $\vartheta, \vartheta+v \in \mathbb{R} \backslash\{\ldots,-2,-1\}$, then we have

$$
\Delta_{b}^{-v}(\mathbf{z}-b+\vartheta)^{(\vartheta)}=\frac{\Gamma(\vartheta+1)}{\Gamma(\vartheta+v+1)}(\mathbf{z}-b+\vartheta)^{(\vartheta+v)}
$$

for each $\mathbf{z} \in \mathbb{N}_{b+v}$.

Motivated by the definition of $n$th order forward sum for uncertain sequence $\varsigma_{\mathbf{z}}$, we define the $v$ th order forward sum for uncertain sequence $\varsigma_{\mathbf{z}}$ as follows: 
Definition 2.4 (Lu, Q. et al (2019)). Let $v$ be a positive real number, $b \in \mathbb{R}$, and $\varsigma_{\mathbf{z}}$ be an uncertain sequence indexed by $\mathbf{z} \in \mathbb{N}_{b}$. Then

$$
\Delta_{b}^{-v} S_{\mathbf{z}}=\frac{1}{\Gamma(v)} \sum_{r=b}^{\mathbf{z}-v}(\mathbf{z}-\sigma(r))^{(v-1)} \boldsymbol{S}_{r}
$$

is called $v$ th order forward fractional sum of uncertain sequence $\boldsymbol{\varsigma}_{\mathbf{z}}$, where $\sigma(r)=r+1$.

Definition 2.5 (Lu, Q. et al (2019)). The fractional Riemann-Liouville-like forward difference for uncertain sequence $\varsigma_{\mathbf{z}}$ is defined by

$$
\Delta_{b}^{\vartheta} \varsigma_{\mathbf{z}}=\Delta^{n}\left(\Delta_{b}^{-(n-\vartheta)} \varsigma_{\mathbf{z}}\right) .
$$

where $v>0$ and $0 \leq n-1<\vartheta \leq n, n$ represents a positive integer.

\section{Uncertain Fractional Forward Difference Equation}

In this section, a definition of the GUFFDE will be exhibited. The analytic solutions to a class of the linear uncertain Riemann-Liouville fractional difference equations will be provided along with ones for linear uncertain first order forward difference equations.

Definition 3.1. A fractional difference equation is called an uncertain fractional difference equation if it is driven by an uncertain sequence. Further, an uncertain fractional forward difference equation for Riemann-Liouville type is the uncertain Riemann-Liouville fractional forward difference equation.

Consider the following generalized Riemann-Liouville fractional difference equation:

$$
\Delta_{\vartheta-n}^{\vartheta} \Upsilon(\mathbf{z})=G(\mathbf{z}+\vartheta-n, \Upsilon(\mathbf{z}+\vartheta-n))+H(\mathbf{z}+\vartheta-n, \Upsilon(\mathbf{z}+\vartheta-n)) \varsigma_{\mathbf{z}+\vartheta-n},
$$

subject to the initial condition

$$
\left.\Delta_{\vartheta-n}^{\vartheta-n+i} \Upsilon(\mathbf{z})\right|_{t=0}=\Upsilon_{i}, \quad i=0,1, \ldots, n-1,
$$

where $\Delta_{\vartheta-\eta}^{\vartheta}$ denotes fractional Riemann-Liouville forward difference with $0 \leq n-1<\vartheta \leq n, G, H$ are two real-valued functions defined on $[0, \infty] \times \mathbb{R}, \mathbf{z} \in \mathbb{N}_{0} \cap[0, T], n \in \mathbb{N}_{0}, \Upsilon_{i} \in \mathbb{R}$ is a crisp number, and $\varsigma_{\vartheta-n}, \varsigma_{\vartheta}, \cdots, \varsigma_{\mathbf{z}+\vartheta-n}$ are i.i.d. uncertain variables with symmetrical uncertainty distribution $\mathcal{L}(a, b)$.

Apply the operator $\Delta_{0}^{-\vartheta}$ to equation (8) and in view of Definition 2.1, Lemma 2.2 and Lemma 2.3, the initial value problem (8) and (9) is equivalent to the following uncertain fractional sum equation:

$$
\begin{aligned}
\Upsilon(\mathbf{z}) & =\sum_{i=0}^{n-1} \frac{\mathbf{z}^{(\vartheta-n+i)}}{\Gamma(\vartheta-n+i+1)} \Upsilon_{i}+\frac{1}{\Gamma(\vartheta)} \sum_{r=0}^{\mathbf{z}-\vartheta}(\mathbf{z}-\sigma(r))^{(\vartheta-1)} \\
& \times\left[G(r+\vartheta-n, \Upsilon(r+\vartheta-n))+H(r+\vartheta-n, \Upsilon(r+\vartheta-n)) \varsigma_{r+\vartheta-n}\right]
\end{aligned}
$$

for $\mathbf{z} \in \mathbb{N}_{\vartheta} \cap[0, T]$ and $n \in \mathbb{N}_{0}$.

In this paper, the following special linear GUFFDE will be considered:

$$
\begin{aligned}
& \left.\Delta_{\vartheta-n}^{\vartheta} \Upsilon(\mathbf{z})=\lambda \Upsilon(\mathbf{z}+\vartheta-n)\right)+\lambda \varsigma_{\mathbf{z}+\vartheta-n}, \\
& \left.\Delta_{\vartheta-n}^{\vartheta-n+i} \Upsilon(\mathbf{z})\right|_{t=0}=\Upsilon_{i}, \quad i=0,1, \ldots, n-1
\end{aligned}
$$

for $\mathbf{z} \in \mathbb{N}_{0} \cap[0, T], n \in \mathbb{N}_{0}$ and $\lambda \in(0,1)$.

Theorem 3.1. The linear GUFFDE (11) subject to the initial condition (12) has a solution

$$
\Upsilon(\mathbf{z})=\Upsilon_{i} F_{\vartheta, \lambda}(\mathbf{z})+\varsigma_{\mathbf{z}}, \quad, i=0,1, \ldots, n-1,
$$

for $\mathbf{z} \in \mathbb{N}_{\vartheta} \cap[0, T], n \in \mathbb{N}_{0}$ and $\lambda \in(0,1)$, where $\varsigma_{\mathbf{z}}$ is an uncertain sequence with the uncertainty distribution $\mathcal{L}\left(a \cdot e_{\vartheta, \lambda}(\mathbf{z}), b \cdot e_{\vartheta, \lambda}(\mathbf{z})\right)$, and

$$
F_{\vartheta, \lambda}(\mathbf{z})=\sum_{k=0}^{\infty} \lambda^{k} \sum_{i=0}^{n-1} \frac{(\mathbf{z}+k(\vartheta-n))^{((k+1) \vartheta-n+i)}}{\Gamma((k+1) \vartheta-n+i+1)},
$$


and

$$
e_{\vartheta, \lambda}(\mathbf{z})=\sum_{k=1}^{\infty} \lambda^{k} \frac{(\mathbf{z}+(k-1)(\vartheta-n))^{(k \vartheta)}}{\Gamma(k \vartheta+1)}
$$

Proof. Apply the operator $\Delta_{0}^{-\vartheta}$ to equation (11) to obtain

$$
\left.\Delta_{0}^{-\vartheta}\left(\Delta_{\vartheta-n}^{\vartheta} \Upsilon(\mathbf{z})\right)=\lambda \Delta_{0}^{-\vartheta} \Upsilon(\mathbf{z}+\vartheta-n)\right)+\lambda \Delta_{0}^{-\vartheta} \varsigma_{\mathbf{z}+\vartheta-n}, \quad \mathbf{z} \in \mathbb{N}_{\vartheta} \cap[0, T] .
$$

By using Lemma 2.2 and Lemma 2.3 to the left-side of (16) to obtain

$$
\begin{aligned}
\Delta_{0}^{-\vartheta}\left(\Delta_{\vartheta-n}^{\vartheta} \Upsilon(\mathbf{z})\right) & =\Delta_{0}^{-\vartheta}\left(\Delta_{0} \Delta_{\vartheta-n}^{\vartheta-n} \Upsilon(\mathbf{z})\right) \\
& =\Delta_{0}\left(\Delta_{0}^{-\vartheta} \Delta^{\vartheta-n} \Upsilon(\mathbf{z})\right)-\sum_{i=0}^{n-1} \frac{\mathbf{z}^{(\vartheta-n+i)}}{\Gamma(\vartheta-n+i+1)} \Upsilon_{i} \\
& =\Upsilon(\mathbf{z})-\sum_{i=0}^{n-1} \frac{\mathbf{z}^{(\vartheta-n+i)}}{\Gamma(\vartheta-n+i+1)} \Upsilon_{i}, \quad \mathbf{z} \in \mathbb{N}_{\vartheta} \cap[0, T] .
\end{aligned}
$$

Then equation (16) becomes

$$
\left.\Upsilon(\mathbf{z})=\sum_{i=0}^{n-1} \frac{\mathbf{z}^{(\vartheta-n+i)}}{\Gamma(\vartheta-n+i+1)} \Upsilon_{i}+\lambda \Delta_{0}^{-\vartheta} \Upsilon(\mathbf{z}+\vartheta-n)\right)+\lambda \Delta_{0}^{-\vartheta} \varsigma_{\mathbf{z}+\vartheta-n}, \quad \mathbf{z} \in \mathbb{N}_{\vartheta} \cap[0, T]
$$

which is the solution of the UFFDE (13).

To drive the solution, we use the Picard approximation by setting $\Upsilon_{0}(\mathbf{z})=\sum_{i=0}^{n-1} \frac{\mathbf{z}^{(\vartheta-n+1)}}{\Gamma(\vartheta-n+i+1)} \Upsilon_{i}$ for $\mathbf{z} \in \mathbb{N}_{\vartheta-n} \cap[0, T]$. The other components can be determined by using the following recurrence relation:

$$
\Upsilon_{j}(\mathbf{z})=\sum_{i=0}^{n-1} \frac{\mathbf{z}^{(\vartheta-n+i)}}{\Gamma(\vartheta-n+i+1)} \Upsilon_{i}+\lambda \Delta_{0}^{-\vartheta} \Upsilon_{j-1}(\mathbf{z}+\vartheta-n)+\lambda \Delta_{0}^{-\vartheta} \varsigma_{\mathbf{z}+\vartheta-n}
$$

for $\mathbf{z} \in \mathbb{N}_{\vartheta} \cap[0, T]$ and $n, j \in \mathbb{N}_{0}$. Since $\varsigma_{\vartheta-n}, \varsigma_{\vartheta}, \cdots, \varsigma_{\mathbf{z}+\vartheta-n}$ are i.i.d. uncertain variables, we write $\varsigma_{\mathbf{z}+\vartheta-n}=\varsigma_{\text {in distribu- }}$ tion. By using Lemma 2.6 and the fact that the linear combination of finite independent uncertain variables (for example, linear uncertain variables, zigzag uncertain variables, normal uncertain variables, and lognormal uncertain variables) is an uncertain variable with positive linear combination coefficient by Theorem 1.21-1.24 of Wu, G., \& Baleanu, D. (2015), we obtain

$$
\begin{aligned}
\Upsilon_{1}(\mathbf{z}) & =\sum_{i=0}^{n-1} \frac{\mathbf{z}^{(\vartheta-n+i)}}{\Gamma(\vartheta-n+i+1)} \Upsilon_{i}+\lambda \Delta_{0}^{-\vartheta} \Upsilon_{0}(\mathbf{z}+\vartheta-n)+\lambda \Delta_{0}^{-\vartheta} \varsigma \\
& =\sum_{i=0}^{n-1} \frac{\mathbf{z}^{(\vartheta-n+i)}}{\Gamma(\vartheta-n+i+1)} \Upsilon_{i}+\lambda \sum_{i=0}^{n-1} \frac{(\mathbf{z}+\vartheta-n)^{(2 \vartheta-n+i)}}{\Gamma(2 \vartheta-n+i+1)} \Upsilon_{i}+\lambda \frac{\mathbf{z}^{(\vartheta)}}{\Gamma(\vartheta+1)} \varsigma \\
\Upsilon_{2}(\mathbf{z}) & =\sum_{i=0}^{n-1} \frac{\mathbf{z}^{(\vartheta-n+i)}}{\Gamma(\vartheta-n+i+1)} \Upsilon_{i}+\lambda \Delta_{0}^{-\vartheta} \Upsilon_{1}(\mathbf{z}+\vartheta-n)+\lambda \Delta_{0}^{-\vartheta} \varsigma, \\
& =\sum_{i=0}^{n-1} \frac{\mathbf{z}^{(\vartheta-n+i)}}{\Gamma(\vartheta-n+i+1)} \Upsilon_{i}+\lambda \sum_{i=0}^{n-1} \frac{(\mathbf{z}+\vartheta-n)^{(2 \vartheta-n+i)}}{\Gamma(2 \vartheta-n+i+1)} \Upsilon_{i}+\lambda^{2} \sum_{i=0}^{n-1} \frac{(\mathbf{z}+2(\vartheta-n))^{(3 \vartheta-n+i)}}{\Gamma(3 \vartheta-n+i+1)} \Upsilon_{i} \\
& +\lambda \frac{\mathbf{z}^{(\vartheta)}}{\Gamma(\vartheta+1)} \varsigma+\lambda^{2} \frac{(\mathbf{z}+\vartheta-n)^{(2 \vartheta)}}{\Gamma(2 \vartheta+1)} \varsigma,
\end{aligned}
$$

and so on. Therefore,

$$
\Upsilon_{j}(\mathbf{z})=\sum_{k=1}^{j} \lambda^{k} \frac{(\mathbf{z}+(k-1)(\vartheta-n))^{(k \vartheta)}}{\Gamma(k \vartheta+1)} \varsigma+\sum_{k=0}^{j} \lambda^{k} \sum_{i=0}^{n-1} \frac{(\mathbf{z}+k(\vartheta-n))^{((k+1) \vartheta-n+i)}}{\Gamma((k+1) \vartheta-n+i+1)} \Upsilon_{i}
$$


for $\mathbf{z} \in \mathbb{N}_{\vartheta} \cap[0, T]$ and $n \in \mathbb{N}_{0}$. Since the two series

$$
\sum_{k=1}^{\infty} \lambda^{k} \frac{(\mathbf{z}+(k-1)(\vartheta-n))^{(k \vartheta)}}{\Gamma(k \vartheta+1)}
$$

and

$$
\sum_{k=0}^{\infty} \lambda^{k} \sum_{i=0}^{n-1} \frac{(\mathbf{z}+k(\vartheta-n))^{((k+1) \vartheta-n+i)}}{\Gamma((k+1) \vartheta-n+i+1)}
$$

are absolutely convergent for $|\lambda|<1$ by the d'Alembert ratio comparison test, the limitation $\bar{Y}(\mathbf{z}):=\lim _{j \rightarrow \infty} \Upsilon_{j}$ exists. Thus

$$
\bar{Y}(\mathbf{z})=\sum_{k=1}^{\infty} \lambda^{k} \frac{(\mathbf{z}+(k-1)(\vartheta-n))^{(k \vartheta)}}{\Gamma(k \vartheta+1)} \varsigma+\sum_{k=0}^{\infty} \lambda^{k} \sum_{i=0}^{n-1} \frac{(\mathbf{z}+k(\vartheta-n))^{((k+1) \vartheta-n+i)}}{\Gamma((k+1) \vartheta-n+i+1)} \Upsilon_{i}, \quad \mathbf{z} \in \mathbb{N}_{\vartheta} \cap[0, T]
$$

Taking limit on both sides of (18) yields

$$
\bar{Y}(\mathbf{z})=\sum_{i=0}^{n-1} \frac{\mathbf{z}^{(\vartheta-n+i)}}{\Gamma(\vartheta-n+i+1)} \Upsilon_{i}+\lambda \Delta_{0}^{-\vartheta} \bar{Y}(\mathbf{z}+\vartheta-n)+\lambda \Delta_{0}^{-\vartheta} \boldsymbol{S}_{\mathbf{z}+\vartheta-n}, \quad \mathbf{z} \in \mathbb{N}_{\vartheta} \cap[0, T], n \in \mathbb{N}_{0} .
$$

That is, $\bar{Y}(\mathbf{z})$ satisfies equation (17). Hence $\bar{Y}(\mathbf{z})$ is a solution of equation (11) subject to the initial condition (12). Thus the proof of Theorem 3.1 is completed.

\section{Existence and Uniqueness}

To find out the conditions that GUFFDEs have solutions, an existence and uniqueness theorem is provided in this section.

Theorem 4.1. Assume that $G(\mathbf{z}, x)$ and $H(\mathbf{z}, x)$ satisfy the Lipschitz condition

$$
|G(\mathbf{z}, x)-G(\mathbf{z}, y)|+|H(\mathbf{z}, x)-H(\mathbf{z}, y)| \leq L|x-y|,
$$

for some positive number $L$ that satisfies the following inequality:

$$
L<\frac{\Gamma(\vartheta+1) \Gamma(T+1-\vartheta)}{\Gamma(T+1)(Q+1)},
$$

where $Q=|a| \vee|b|$. Then GUFFDE (11) subject to the initial condition (12) has a unique solution $\Upsilon(\mathbf{z})$ for $\mathbf{z} \in \mathbb{N}_{\vartheta} \cap[0, T]$ almost surely.

Proof. Let $l_{\vartheta}^{k}$ be the set of all finite real sequences $x=\{x(\mathbf{z})\}_{\vartheta}^{k}$ which has $k$ terms with the norm $x=\max _{\mathbf{z} \in \mathbb{N}_{\vartheta} \cap[0, T]}|x(\mathbf{z})|$. It is evident that $\left(l_{\vartheta}^{k},\|\|.\right)$ is a Banach space. For any $\chi_{\mathbf{z}}, \Upsilon_{\mathbf{z}} \in l_{\vartheta}^{k}$, the operator $P$ is defined as follows:

$$
\begin{aligned}
P \chi_{\mathbf{z}} & =\sum_{i=0}^{n-1} \frac{\mathbf{z}^{(\vartheta-n+i)}}{\Gamma(\vartheta-n+i+1)} \Upsilon_{i}+\frac{1}{\Gamma(\vartheta)} \sum_{r=0}^{\mathbf{z}-\vartheta}(\mathbf{z}-\sigma(r))^{(\vartheta-1)} \\
& \times\left[G(r+\vartheta-n, \Upsilon(r+\vartheta-n))+H(r+\vartheta-n, \Upsilon(r+\vartheta-n)) \varsigma_{r+\vartheta-n}\right] .
\end{aligned}
$$

At each time $t$, since $\varsigma_{\mathbf{z}}\left(\mathbf{z} \in \mathbb{N}_{\vartheta} \cap[0, T]\right)$ is an uncertain variable who has linear uncertainty distribution $\mathcal{L}(a, b)$, we have $\mathcal{M}\left\{\left(\varsigma_{\mathbf{z}}<a\right) \cup\left(\varsigma_{\mathbf{z}}>b\right)\right\}=0$. For any given $\gamma \in \chi\left\{\left(\varsigma_{\mathbf{z}}<a\right) \cup\left(\varsigma_{\mathbf{z}}>b\right)\right\}, \varsigma_{\mathbf{z}}(\gamma) \leq Q(Q=|a| \vee|b|)$ holds almost surely for 
$\mathbf{z} \in \mathbb{N}_{\vartheta} \cap[0, T]$, where $\chi$ is the universal set on the uncertainty space. Then by the hypothesis and Lemma 2.4 , we have

$$
\begin{aligned}
\left\|P \chi_{\mathbf{z}}(\gamma)-P \Upsilon_{\mathbf{z}}(\gamma)\right\| & =\max _{\mathbf{z} \in \mathbb{N}_{\vartheta} \cap[0, T]}\left|P \chi_{\mathbf{z}}(\gamma)-P \Upsilon_{\mathbf{z}}(\gamma)\right| \\
& \leq \frac{1}{\Gamma(\vartheta)} \max _{\mathbf{z} \in \mathbb{N}_{\vartheta} \cap[0, T]} \sum_{r=0}^{\mathbf{z}-\vartheta}(\mathbf{z}-\sigma(r))^{(\vartheta-1)} \\
& \times\left(\mid G\left(r+\vartheta-n, \chi_{r+\vartheta-n}(\gamma)-G\left(r+\vartheta-n, \Upsilon_{r+\vartheta-n}(\gamma) \mid\right.\right.\right. \\
& +\mid\left[H\left(r+\vartheta-n, \chi_{r+\vartheta-n}(\gamma)-H\left(r+\vartheta-n, \Upsilon_{r+\vartheta-n}(\gamma)\right] \varsigma_{r+\vartheta-n} \mid\right)\right. \\
& \leq \frac{1}{\Gamma(\vartheta)} \max _{\mathbf{z} \in \mathbb{N}_{\vartheta} \cap[0, T]} \sum_{r=0}^{\mathbf{z}-\vartheta}(\mathbf{z}-\sigma(r))^{(\vartheta-1)} \\
& \times\left(\mid G\left(r+\vartheta-n, \chi_{r+\vartheta-n}(\gamma)-G\left(r+\vartheta-n, \Upsilon_{r+\vartheta-n}(\gamma) \mid\right.\right.\right. \\
& +Q \mid H\left(r+\vartheta-n, \chi_{r+\vartheta-n}(\gamma)-H\left(r+\vartheta-n, \Upsilon_{r+\vartheta-n}(\gamma) \mid\right)\right. \\
& \leq L(1+Q) \frac{1}{\Gamma(\vartheta)} \max _{\mathbf{z} \in \mathbb{N}_{\vartheta} \cap[0, T]} \sum_{r=0}^{\mathbf{z}-\vartheta}(\mathbf{z}-\sigma(r))^{(\vartheta-1)}\left|\chi_{r+\vartheta-n}(\gamma)-\Upsilon_{r+\vartheta-n}(\gamma)\right| \\
& \leq L(1+Q)\left\|\chi_{\mathbf{z}}(\gamma)-\Upsilon_{\mathbf{z}}(\gamma)\right\| \max _{\mathbf{z} \in \mathbb{N}_{\vartheta} \cap[0, T]}\left(\Delta_{0}^{-\vartheta} \mathbf{z}^{(0)}\right) \\
& =L(1+Q)\left\|\chi_{\mathbf{z}}(\gamma)-\Upsilon_{\mathbf{z}}(\gamma)\right\| \max _{\mathbf{z} \in \mathbb{N}_{\vartheta} \cap[0, T]}\left(\frac{1}{\Gamma(\vartheta+1)} \mathbf{z}^{(\vartheta)}\right) \\
& \leq \frac{L(1+Q) T^{(\vartheta)}}{\Gamma(\vartheta+1)}\left\|\chi_{\mathbf{z}}(\gamma)-\Upsilon_{\mathbf{z}}(\gamma)\right\| \\
& =\frac{L(1+Q) \Gamma(T+1)}{\Gamma(\vartheta+1) \Gamma(T-\vartheta+1)}\left\|\chi_{\mathbf{z}}(\gamma)-\Upsilon_{\mathbf{z}}(\gamma)\right\| . \\
& a(0)
\end{aligned}
$$

With $0<L<\frac{\Gamma(\vartheta+1) \Gamma(T-\vartheta+1)}{(1+Q) \Gamma(T+1)}, P$ is a contraction mapping in $l_{\vartheta}^{k}$ almost surely. Thus we obtain a unique fixed point $\Upsilon_{\mathbf{z}}(\gamma)$ of $P$ in $l_{\vartheta}^{k}$ almost surely by the Banach contraction mapping theorem. Furthermore, $\Upsilon_{\mathbf{z}}(\gamma)=\lim _{j \rightarrow \infty} \Upsilon_{\mathbf{z}}^{j}(\gamma)$, where $\Upsilon_{\mathbf{z}}^{j}(\gamma)=P\left(\Upsilon_{\mathbf{z}}^{j-1}(\gamma)\right)$ with $\Upsilon_{\mathbf{z}}^{0}(\gamma)=\sum_{i=0}^{n-1} \frac{\mathbf{z}^{(\vartheta-n+i)}}{\Gamma(\vartheta-n+i+1)} \Upsilon_{i}$.

For any given $\mathbf{z} \in \mathbb{N}_{\vartheta} \cap[0, T]$, as $G$ and $H$ are Lipschitz continuous functions, the operator $P$ is measurable. Note that $\Upsilon_{\mathbf{z}}^{0}(\gamma)$ is an uncertain variable, $\Upsilon_{\mathbf{z}}^{1}(\gamma), \Upsilon_{\mathbf{z}}^{2}(\gamma), \ldots, \Upsilon_{\mathbf{z}}^{j}(\gamma), \ldots$ are uncertain variables since a real-valued measurable function of uncertain variables is an uncertain variable by Theorem 1.10 in Wu, G., \& Baleanu, D. (2015). Thus $\Upsilon_{\mathbf{z}}=\lim _{j \rightarrow \infty} \Upsilon_{\mathbf{z}}^{j}$ is an uncertain variable by Theorem 3 in Zhu, Y. (2015b).

Consequently, UFFDE (11) subject to the initial condition (12) has a unique solution $\Upsilon_{\mathbf{z}}$ for $\mathbf{z} \in \mathbb{N}_{\vartheta} \cap[0, T]$ almost surely.

\section{Test Examples}

In this section, we present two examples to illustrate Theorem 4.1.

Example 5.1. Consider the following UFFDE:

$$
\Delta_{\pi-4}^{\pi} \Upsilon(\mathbf{z})=\left\{\begin{array}{l}
-\frac{\ln (|\Upsilon(\mathbf{z}+\pi-4)|+1)}{(\mathbf{z}+4)^{5}}+\varsigma_{\mathbf{z}+\pi-4}, \quad \mathbf{z} \in \mathbb{N}_{0} \cap[0,12], \\
\Upsilon(\pi-4)=\Delta \Upsilon(\pi-4)=\Delta^{2} \Upsilon(\pi-4)=0, \\
\Delta_{\pi-4}^{\frac{e}{2}} Y\left(12+\pi-\frac{e}{2}\right)=0,
\end{array}\right.
$$

where $\varsigma_{\pi-4}, \varsigma_{\pi-4+1}, \cdots, \varsigma_{\pi-4+12}$ are i.i.d. uncertain variables with uncertainty distribution $\mathcal{L}(-3,3)$.

According to Theorem 4.1 with $n=4$, the inverse uncertainty distribution of the solution for the UFFDE (21) is the solution of the following sum equation:

$$
\Upsilon(\mathbf{z})=\sum_{i=0}^{3} \frac{\mathbf{z}^{(\pi-4)}}{\Gamma(\pi)} \Upsilon_{i}+\frac{1}{\Gamma(\pi)} \sum_{r=0}^{\mathbf{z}-\pi}(\mathbf{z}-\sigma(r))^{(\pi-4)}\left(\frac{\ln (|\Upsilon(r+\pi-4)|+1)}{(r+4)^{5}}+\varsigma_{r+4}\right)
$$


With $\vartheta \in(3,4]$, it can be easily verified that

$$
\begin{aligned}
|G(\mathbf{z}, x)-G(\mathbf{z}, y)|+|H(\mathbf{z}, x)-H(\mathbf{z}, y)| & =\left|\frac{\ln (|x|+1)}{(\mathbf{z}+4)^{5}}-\frac{\ln (|y|+1)}{(\mathbf{z}+4)^{5}}\right| \\
& =\frac{1}{(\mathbf{z}+4)^{5}}|\ln (|x|+1)-\ln (|y|+1)| \\
& \leq \frac{1}{4^{5}} \| x|-| y|| \\
& \leq \frac{|x-y|}{1024},
\end{aligned}
$$

and

$$
\frac{\Gamma(\pi+1) \Gamma(12+1-\pi)}{3 \Gamma(12+1)} \approx 0.0013>\frac{1}{24}=0.00098 .
$$

Thus, UFFDE (21) has a unique solution almost surely by Theorem 4.1.

Example 5.2. Consider the following UFFDE:

$$
\Delta_{-0.5}^{0.5} \Upsilon(\mathbf{z})=\frac{\sin \Upsilon(\mathbf{z}-0.5)}{10+(\mathbf{z}-0.5)^{2}}+0.1 \varsigma_{\mathbf{z}-0.5}, \quad \mathbf{z} \in \mathbb{N}_{0} \cap[0,3],
$$

where $\varsigma_{-0.5}, \varsigma_{0.5}, \varsigma_{1.5}, \varsigma_{2.5}$ are 4 i.i.d. linear uncertain variables with linear uncertainty distribution $\mathcal{L}(-1,1)$.

According to Theorem 4.1 with $n=1$, the inverse uncertainty distribution of the solution for the UFFDE (22) is the solution of the following sum equation:

$$
\Upsilon(\mathbf{z})=\frac{1}{\sqrt{\pi \mathbf{z}}} \Upsilon_{0}+\frac{1}{\sqrt{\pi}} \sum_{r=0}^{\mathbf{z}-\frac{1}{2}}(\mathbf{z}-\sigma(r))^{\left(-\frac{1}{2}\right)}\left(\frac{\sin \Upsilon(r-0.5)}{10+(r-0.5)^{2}}+0.1 \varsigma_{r-0.5}\right) .
$$

It can be easily verified that

$$
|G(\mathbf{z}, x)-G(\mathbf{z}, y)|+|H(\mathbf{z}, x)-H(\mathbf{z}, y)| \leq \frac{1}{10}|x-y|,
$$

and

$$
\frac{\Gamma(0.5+1) \Gamma(3+1-0.5)}{2 \Gamma(3+1)} \approx 0.2454>\frac{1}{10}=0.1 .
$$

Then UFFDE (22) has a unique solution almost surely by Theorem 4.1 .

\section{References}

Agarwal, R. P. (2000). Difference Equations and Inequalities: Theory, Methods, and Application. Marcel Dekker, New York, NY, USA.

Atici, F., \& Eloe, P. (2007). A transform method in discrete fractional calculus. Integral and Finite Difference Inequalities and Applications, 2, 165-176.

Atici, F., \& Eloe, P. (2009). Initial value problems in discrete fractional calculus, Proceeding of American Mathematical Society, 137, 981-989.

Bastos, N. R. O., Ferreira, R. A. C., \& Torres, D. F. M. (2011). Discrete-time fractional variational problems. Signal Process, 91(3), 513-524. https://doi.org/10.1016/j.sigpro.2010.05.001

Bohner, M., \& Peterson, A. C. (2003). Advances in Dynamic Equations on Time Scales. Birkhauser, Boston, Mass, USA. https://doi.org/10.1007/978-0-8176-8230-9

Diethelm, K. (2010). The Analysis of Fractional Differential Equations. Springer, Berlin, Germany. https://doi.org/10.1007/978-3-642-14574-2

Goodrich, C. (2011). Existence of a positive solution to a system of discrete fractional boundary value problems. Applied Mathematics and Computation, 217, 4740-4753. https://doi.org/10.1016/j.amc.2010.11.029 
Goodrich, C., \& Peterson, A. (2015). Discrete Fractional Calculus. Springer, Berlin. https://doi.org/10.1007/978-3-31925562-0

Hamasalh, F. K., \& Mohammed, P. O. (2015a). Generalized quartic fractional spline approximation function with applications. Int. J. Open Problems Compt. Math., 8(1), 67-80.

Hamasalh, F. K., \& Mohammed, P. O. (2015b). Numerical Solution of Fractional Differential Equations by using Fractional Spline Model. J. Information and Computing Science, 10(2), 098-105. https://doi.org/10.17656/jzs.10404

Kilbas, A. A., Srivastava, H. M., \& Trujillo, J. J. (2006). Theory and Applications of Fractional Differential Equations. Elsevier B.V., Amsterdam, Netherlands. https://doi.org/10.3182/20060719-3-PT-4902.00008

Holm, M. (2011). The theory of discrete fractional calculus: development and application. PhD Dissertation, University of Nebraska-Lincoln.

Liu, B. (2010). Uncertainty Theory: A Branch of Mathematics for Modeling Human Uncertainty. Springer, Berlin.

Lu, Z., \& Zhu, Y. (2018). Comparison principles for fractional differential equations with the Caputo derivatives. Advances in Difference Equations, 237, 1-11. https://doi.org/10.1186/s13662-018-1691-y

Lu, Z., \& Zhu, Y. (2019). Numerical approach for solution to an uncertain fractional differential equation. Applied Mathematics and Computation, 343, 137-148. https://doi.org/10.1016/j.amc.2018.09.044

Lu, Q., Zhu, Y., \& Lu, Z. (2019). Uncertain fractional forward difference equations for Riemann-Liouville type. Advances in Difference Equations, 2019, 147. https://doi.org/10.1186/s13662-019-2093-5

Miller, K. S., \& Ross, B. (1993). An Introduction to the Fractional Calculus and Fractional Differential Equations. John Wiley \& Sons, New York, NY, USA.

Podlubny, I. (1999). Fractional Differential Equations. Academic Press, San Diego, Calif, USA.

Wu, G., \& Baleanu, D. (2015). Discrete chaos in fractional delayed logistic maps. Nonlinear Dynamics, 80(4), 1697-1703. https://doi.org/10.1007/s11071-014-1250-3

Wu, G., Baleanu, D., \& Luo, W. (2017). Lyapunov functions for Riemann-Liouville-like fractional difference equations. Applied Mathematics and Computation, 314, 228-236. https://doi.org/10.1016/j.amc.2017.06.019

Wu, G., Baleanu, D., \& Zeng, S. (2018). Finite-time stability of discrete fractional delay systems: Gronwall inequality and stability criterion. Communications in Nonlinear Science and Numerical Simulation, 57, 299-308. https://doi.org/10.1016/j.cnsns.2017.09.001

Zhu, Y. (2015a). Uncertain fractional differential equations and an interest rate model. Mathematical Methods in the Applied Sciences, 38, 3359-3368.

Zhu, Y. (2015b). Existence and uniqueness of the solution to uncertain fractional differential equation. Journal of Uncertainty Analysis and Applications, 3, 1-11. https://doi.org/10.1186/s40467-015-0028-6

\section{Copyrights}

Copyright for this article is retained by the author(s), with first publication rights granted to the journal.

This is an open-access article distributed under the terms and conditions of the Creative Commons Attribution license (http://creativecommons.org/licenses/by/4.0/). 